Transactions of Karelian Research Centre RAS

No. 7. 2018. P. $140-148$

DOI: $10.17076 / \operatorname{mat769}$
Труды Карельского научного центра РАН

№ 7. 2018. C. $140-148$

УДК 519.179.4

\title{
LIMIT DISTRIBUTIONS OF VERTEX DEGREES IN A CONDITIONAL CONFIGURATION GRAPH
}

\author{
I. A. Chepliukova, Yu. L. Pavlov \\ Institute of Applied Mathematical Research of the Karelian Research Centre \\ of the Russian Academy of Sciences, Petrozavodsk, Russia
}

\begin{abstract}
The configuration graph where vertex degrees are independent identically distributed random variables is often used for modeling of complex networks such as the Internet. We consider a random graph consisting of $N$ vertices. The random variables $\eta_{1}, \ldots, \eta_{N}$ are equal to the degrees of vertices with the numbers $1, \ldots, N$. The probability $\mathbf{P}\left\{\eta_{i}=k\right\}, i=1, \ldots, N, k=1,2, \ldots$, is equivalent to $h(k) / k^{\tau}$ as $k \rightarrow \infty$, where $h(x)$ is a slowly varying function integrable in any finite interval, $\tau>1$. We obtain the limit distributions of the maximum vertex degree and the number of vertices with a given degree under the condition that the sum of degrees is equal to $n$ and $N, n \rightarrow \infty$.
\end{abstract}

K e y w ords: configuration graph; the limit distribution; vertex degree.

\section{И. А. Чеплюкова, Ю. Л. Павлов. ПРЕДЕЛЬНЫЕ РАСПРЕ- ДЕЛЕНИЯ СТЕПЕНЕЙ ВЕРШИН В УСЛОВНОМ КОНФИ- ГУРАЦИОННОМ ГРАФЕ}

Конфигурационный граф, степени вершин которого являются независимыми одинаково распределенными случайными величинами, часто используют для моделирования сложных сетей, таких как Интернет. Мы рассматриваем случайный граф с $N$ вершинами. Случайные величины $\eta_{1}, \ldots, \eta_{N}$ равны степеням вершин с номерами $1, \ldots, N$. Вероятность $\mathbf{P}\left\{\eta_{i}=k\right\}, i=1, \ldots, N, k=1,2, \ldots$, пропорциональна величине $h(k) / k^{\tau}$ при $k \rightarrow \infty$, где $h(x)$ - интегрируемая на любом конечном интервале медленно меняющаяся функция и $\tau>1$. Найдены предельные распределения максимальной степени вершин и числа вершин заданной степени при условии, что сумма степеней равна $n$ при $N, n \rightarrow \infty$.

К л ю ч е в ы е с л о в а: конфигурационный граф; предельное распределение; степень вершины.

\section{INTRODUCTION}

The study of random graphs has been causing growing interest in connection with the wide use of these models for the description of complex networks (see, e. g.[3, 6, 11]. Such models can be used to adequate by describe the topology of transport, electricity, social, telecommunication networks and global Internet. Observations on real networks showed that their topology can be described by random graphs with vertex degrees being independent identically distributed random variables with power-law distribution. In [3] it was suggested that for large $k$ the number of vertices with the degree $k$ is proportional to $k^{-\tau}$, where $\tau>1$. That is why in [11] it was suggested that the distribution of the vertex degree $\eta$ is 


$$
\mathbf{P}\{\eta \geqslant k\}=h(k) k^{-\tau+1}, \quad k=1,2, \ldots,
$$

where $h(k)$ is a slowly varying function.

We consider a random graph consisting of $N+1$ vertices. Let random variables $\eta_{1}, \ldots, \eta_{N}$ be equal to the degrees of vertices with the numbers $1, \ldots, N$. Each vertex is assigned a certain degree in accordance with the distribution (1). The vertex degree is the number of stubs (or semiedges) that are numbered in an arbitrary order. Stubs are vertex edges for which adjacent nodes are not yet determined. The vertex 0 is auxiliary and has degree 0 if the sum of all other vertices is even, else the degree is 1 . It is clear that we need to use the auxiliary vertex 0 for the sum of degrees to be even. The graph is constructed by joining all the stubs pairwise equiprobably to form links.

There are many papers where the results describing the limit behaviour of different random graph characteristics were obtained. The authors of [11] were sure (without proof) that the function $h(k)$ in (1) does not influence the limit results, and that to study the configuration graph one can replace $h(k)$ with the constant 1 . In our work we will show that the role of the slowly varying function $h(k)$ is more complicated.

We consider the subset of random graphs under the condition $\eta_{1}+\cdots+\eta_{N}=n$. Such conditional graphs can be useful for modeling of networks for which we can estimate the number of communications. They are useful also for studying networks without conditions on the number of links by averaging the results of conditional graphs with respect to the distribution of the sum of degrees. Conditional random graphs were first analyzed in [9], where $h(k) \equiv 1$. Obviosly, the limit behaviour of a random graph depends on the degree structure. In [9] the limit distributions were obtained for the maximum vertex degree and the number of vertices of a given degree as $N$ and $n$ tend to infinity in such a way that $1<n / N<$ $\zeta(\tau)$, where $\zeta(\tau)$ is the value of the Rimman's zeta-function at the point $\tau$. For other zones of parameters analogous results were obtained in papers $[7,8,10]$.

Here we extend the results on the maximum vertex degree and the number of vertices of a given degree to the configuration graphs with degree distribution (1), where $h(k)$ is not constant. In the following section the main results are formulated, then auxiliary statements are proved. And the last section contains proofs of the main results.

\section{Main Results}

In the paper we assume that the distributions of node degrees are

$$
p_{k}=\mathbf{P}\left\{\eta_{i}=k\right\}=\frac{h(k)}{k^{\tau} \Sigma(1, \tau)},
$$

where $i=1, \ldots, N, \quad k=1,2, \ldots, \quad \tau>$ $1, \quad h(k)$ is a slowly varying function integrable in any finite interval and

$$
\Sigma(x, y)=\sum_{k=1}^{\infty} x^{k} h(k) k^{-y} .
$$

We denote also by $\xi_{1}, \ldots, \xi_{N}$ the auxiliary independent identically distributed random variables such that

$$
p_{r}(\lambda)=\mathbf{P}\left\{\xi_{i}=k\right\}=\lambda^{k} p_{k} \Sigma(1, \tau) / \Sigma(\lambda, \tau),
$$

where $i=1, \ldots, N, k=1,2, \ldots$ and the parameter $\lambda=\lambda(n, N)$ belongs to the interval $(0,1)$. From $(2)-(4)$ we obtain

$$
\begin{gathered}
m=\mathbf{E} \xi_{1}=\Sigma(\lambda, \tau-1) / \Sigma(\lambda, \tau), \\
\sigma^{2}=\mathbf{D} \xi_{1}=\Sigma(\lambda, \tau-2) / \Sigma(\lambda, \tau)-m^{2} .
\end{gathered}
$$

Let the parameter $\lambda=\lambda(n, N)$ of the distribution (4) be determined by the relation

$$
m=\Sigma(\lambda, \tau-1) / \Sigma(\lambda, \tau)=n / N .
$$

We denote by $\eta_{(N)}$ and $\mu_{r}$ the maximum vertex degree and the number of vertices with the degree $r$, respectively. We get the following results.

Theorem 1. Let $n, N \rightarrow \infty, n / N \rightarrow$ $1,(n-N)^{3} / N^{2} \rightarrow \infty$ and let $r$ be such that

$$
\frac{N \lambda^{r-1} h(r)}{r^{\tau}} \rightarrow \infty, \quad \frac{N \lambda^{r+1} h(r+1)}{\Sigma(\lambda, \tau)(r+1)^{\tau}} \rightarrow \gamma,
$$

where $\gamma$ is a nonnegative constant. Then

$$
\begin{gathered}
\mathbf{P}\left\{\eta_{(N)}=r\right\} \rightarrow e^{-\gamma}, \\
\mathbf{P}\left\{\eta_{(N)}=r+1\right\} \rightarrow 1-e^{-\gamma} .
\end{gathered}
$$

We introduce the conditions: 


$$
\begin{array}{ll}
\text { (A1) } & \tau>4 ; \\
\text { (A2) } & 3<\tau \leqslant 4, \quad(1-\lambda)^{\tau-4-\varepsilon} / \sqrt{\mathrm{N}} \rightarrow 0 \\
\text { (A3) } & 5 / 2<\tau \leqslant 3, \quad \mathrm{~N}(1-\lambda)^{11-3 \tau+\varepsilon} \geqslant \mathrm{C}_{3}>0 \\
\text { (A4) } & \tau=5 / 2, \quad \mathrm{~N}(-\ln (1-\lambda))^{2}(1-\lambda)^{7 / 2+\varepsilon} \geqslant \mathrm{C}_{4}>0 ; \\
\text { (A5) } & 1<\tau<5 / 2, \quad \mathrm{~N}(1-\lambda)^{6-\tau+\varepsilon} \geqslant \mathrm{C}_{5}>0,
\end{array}
$$

where $\varepsilon$ is some sufficiently small positive constant.

Theorem 2. Let $N, n \rightarrow \infty, n / N \nearrow \Sigma(1, \tau-$ $1) / \Sigma(1, \tau)$, one of the following conditions (A1) - (A5) is fulfilled, and $r=r(N, n)$ take values in such a way that

$$
\frac{N \lambda^{r+1} h(r+1)}{(r+1)^{\tau} \Sigma(\lambda, \tau)(1-\lambda)} \rightarrow \gamma,
$$

where $\gamma$ is a positive constant. Then

$$
\mathbf{P}\left\{\eta_{(N)} \leqslant r\right\}=e^{-\gamma}(1+o(1)) .
$$

Theorem 3. Let $n, N \rightarrow \infty$ and one of the following conditions is fulfilled

1. $n / N \rightarrow 1, \quad r=1,2, \quad(n-N)^{2} / N \rightarrow \infty$;

2. $n / N \rightarrow 1, \quad r \geqslant 3, \quad N \lambda^{r-1} \rightarrow \infty$;

3. $n / N \nearrow \Sigma(1, \tau-1) / \Sigma(1, \tau)$, parameters $\tau, N, n$ are determined by one of the conditions (A1) - (A5).

Then for a nonnegative integer $k$ uniformly with respect to $u=\left(k-N p_{r}(\lambda)\right) /\left(\sigma_{r r} \sqrt{N}\right)$ lies in any fixed finite interval

$$
\mathbf{P}\left\{\mu_{r}=k\right\}=\frac{1}{\sigma_{r r} \sqrt{2 \pi N}} e^{-u^{2} / 2}(1+o(1)),
$$

where

$$
\sigma_{r r}^{2}=p_{r}(\lambda)\left(1-p_{r}(\lambda)-\frac{(n / N-r)^{2}}{\sigma^{2}} p_{r}(\lambda)\right) .
$$

Theorem 4. Let $n, N \rightarrow \infty, n / N \rightarrow 1, n-N \rightarrow$ $\infty, r \geqslant 2$. Then

$\mathbf{P}\left\{\mu_{r}=k\right\}=\frac{1+o(1)}{k !}\left(N p_{r}(\lambda)\right)^{k} \exp \left\{-N p_{r}(\lambda)\right\}$

uniformly with respect to $\left(k-N p_{r}(\lambda)\right) / \sqrt{N p_{r}(\lambda)}$ lies in any fixed finite interval. This assertion remains true for $r \rightarrow \infty$ if $1<n / N<$ $\Sigma(1, \tau-1) / \Sigma(1, \tau)$ under one of the following conditions:

$$
\text { 1. } n / N \rightarrow 1, \quad n-N \rightarrow \infty \text {; }
$$

2. $n / N \nearrow \Sigma(1, \tau-1) / \Sigma(1, \tau)$, parameters $\tau, N, n$ are determined by one of the conditions (A1) - (A5).

Remark. In [2], a case of these theorems under the condition $1<C_{1} \leqslant n / N \leqslant C_{2}<$ $\Sigma(1, \tau-1) / \Sigma(1, \tau)$ was proved.

\section{Auxiliary Results}

We prove some auxiliary statements (Lemmas 1-6), and use them to prove Theorems 1-5. The technique of obtaining these theorems is based on the generalized allocation scheme suggested by V. F. Kolchin [5]. It is readily seen that for our subset of random graphs

$$
\begin{gathered}
\mathbf{P}\left\{\eta_{1}=k_{1}, \ldots, \eta_{N}=k_{N}\right\}= \\
=\mathbf{P}\left\{\xi_{1}=k_{1}, \ldots, \xi_{N}=k_{N} \mid \xi_{1}+\ldots+\xi_{N}=n\right\} .
\end{gathered}
$$

Therefore, the conditions of the generalized allocation scheme are valid (see [5]). Let $\xi_{1}^{(r)}, \ldots, \xi_{N}^{(r)}$ and $\tilde{\xi}_{1}^{(r)}, \ldots, \tilde{\xi}_{N}^{(r)}$ be two sets of independent identically distributed random variables such that

$$
\mathbf{P}\left\{\xi_{1}^{(r)}=k\right\}=\mathbf{P}\left\{\xi_{1}=k \mid \xi_{1} \leqslant r\right\},
$$

$\mathbf{P}\left\{\tilde{\xi}_{1}^{(r)}=k\right\}=\mathbf{P}\left\{\xi_{1}=k \mid \xi_{1} \neq r\right\}, \quad k=1,2, \ldots$

We also put

$$
\begin{gathered}
\zeta_{N}=\xi_{1}+\ldots+\xi_{N}, \quad \zeta_{N}^{(r)}=\xi_{1}^{(r)}+\ldots+\xi_{N}^{(r)}, \\
\tilde{\zeta}_{N}^{(r)}=\tilde{\xi}_{1}^{(r)}+\ldots+\tilde{\xi}_{N}^{(r)}, \quad P_{r}=\mathbf{P}\left\{\xi_{1}>r\right\} .
\end{gathered}
$$

It is shown in [5] that

$$
\begin{gathered}
\mathbf{P}\left\{\eta_{(N)} \leqslant r\right\}=\left(1-P_{r}\right)^{N} \frac{\mathbf{P}\left\{\zeta_{N}^{(r)}=n\right\}}{\mathbf{P}\left\{\zeta_{N}=n\right\}}, \\
\mathbf{P}\left\{\mu_{r}=k\right\}=\left(\begin{array}{c}
N \\
k
\end{array}\right) p_{r}^{k}(\lambda)\left(1-p_{r}(\lambda)\right)^{N-k} \times \\
\\
\times \frac{\mathbf{P}\left\{\tilde{\zeta}_{N-k}^{(r)}=n-k r\right\}}{\mathbf{P}\left\{\zeta_{N}=n\right\}} .
\end{gathered}
$$

From (2)-(6) we can deduce the next lemma.

Lemma 1. Let $N, n \rightarrow \infty$. The next assertions are true: 
1. if $n / N \rightarrow 1$ then $\lambda=\left((n / N-1) p_{1} / p_{2}\right)(1+$ $o(1))$;

2. if $n / N \nearrow \Sigma(1, \tau-1) / \Sigma(1, \tau)$ then $\lambda \rightarrow 1$.

Let us consider the limit behaviour of $\zeta_{N}$.

Lemma 2. Under the conditions of Theorems $1-4$

$$
\mathbf{P}\left\{\zeta_{N}=k\right\}=\frac{1+o(1))}{\sigma \sqrt{2 \pi N}} \exp \left\{-\frac{(k-n)^{2}}{2 \sigma^{2} N}\right\}
$$

uniformly with respect to integers $k$ such that $(k-n) /(\sigma \sqrt{N})$ lies in any fixed finite interval.

Proof. Let $\varphi(t)$ be the characteristic function of the random variable $\xi_{1}$. Then

$$
\varphi(t)=\Sigma\left(e^{i t} \lambda, \tau\right) / \Sigma(\lambda, \tau) .
$$

Further we will need an explicit form of the third derivative of $\ln \varphi(t)$. From (4) it is not hard to get that

$$
\begin{gathered}
(\ln \varphi(t))^{\prime \prime \prime}=i\left(-\frac{\Sigma\left(e^{i t} \lambda, \tau-3\right)}{\Sigma\left(e^{i t} \lambda, \tau\right)}+\right. \\
+3 \frac{\Sigma\left(e^{i t} \lambda, \tau-2\right) \Sigma\left(e^{i t} \lambda, \tau-1\right)}{\Sigma^{2}\left(e^{i t} \lambda, \tau\right)}- \\
\left.-2 \frac{\Sigma^{3}\left(e^{i t} \lambda, \tau-1\right)}{\Sigma^{3}\left(e^{i t} \lambda, \tau\right)}\right) .
\end{gathered}
$$

Let $n / N \rightarrow 1$. From (2)-(4) it is easy to obtain that

$$
\sigma^{2}=O(\lambda), \quad\left|(\ln \varphi(t))^{\prime \prime \prime}\right| \leqslant C_{3} \lambda .
$$

Let $\varphi_{N}(t)$ be the characteristic function of the random variable $\left(\zeta_{N}-n\right) /(\sigma \sqrt{N})$. Then

$$
\begin{aligned}
\ln \varphi_{N}(t) & =-\frac{i n t}{\sigma \sqrt{N}}+N \ln \varphi\left(\frac{t}{\sigma \sqrt{N}}\right)= \\
= & -\frac{t^{2}}{2}+\frac{t^{3} Q(t /(\sigma \sqrt{N}))}{6 \sigma^{3} \sqrt{N}} .
\end{aligned}
$$

Then from Lemma 1, (12) and (13) follows relation

$$
\ln \varphi_{N}(t)=-t^{2} / 2+o(1) .
$$

Let $n / N \nearrow \Sigma(1, \tau-1) / \Sigma(1, \tau)$. It is well known (see e.g. [4]) that the slowly varying function integrable in any finite interval has the following properties:

1. $h(x)>1 / \sqrt{x}$ for sufficiently large $x$;

2. $\quad \lim _{x \rightarrow \infty} h(x+t) / h(x)=1, \quad t \geqslant 0$;

3. $\quad \lim _{x \rightarrow \infty} h(x) / x^{\varepsilon}=0, \quad \lim _{x \rightarrow \infty} h(x) x^{\varepsilon}=\infty$ for any $\varepsilon>0$;

4. $h(x)=c(x) \exp \left\{\int_{\alpha}^{x} \varepsilon(t) / t d t\right\}$, where $\alpha>0, c(x) \rightarrow c \neq 0, \varepsilon(x) \rightarrow 0$, as $x \rightarrow \infty$.

Using the properties (15) and Lemma 1 we can deduce that for $j=0,1,2,3$

$$
\begin{gathered}
\left|\Sigma\left(e^{i t} \lambda, \tau-j\right)\right| \leqslant C_{4} \lambda \Phi(\lambda, \tau-j, 1)+ \\
+\lambda \Phi(\lambda, \tau-j-\varepsilon, 1), \\
\left|\Sigma\left(e^{i t} \lambda, \tau\right)\right| \geqslant C_{5} \quad \text { as } \quad t \rightarrow 0,
\end{gathered}
$$

where $\Phi(x, s, a)$ is the Lerch transcendent function:

$$
\Phi(x, s, a)=\sum_{k=0}^{\infty} x^{k}(k+a)^{-s} .
$$

It is well known (see e.g. [1]) that for the Lerch transcendent function the following properties are valid:

$$
\begin{array}{ll}
\text { 1. } \quad & \lambda \Phi(\lambda, 1,1)=-\ln (1-\lambda), \\
& (1-\lambda) \Phi(\lambda, 0,1)=1 ; \\
2 . \quad & (1-\lambda) \Phi(\lambda, \tau, 1)=O\left((1-\lambda)^{\tau}\right), \\
& \tau<1, \quad \lambda \rightarrow 1 .
\end{array}
$$

From (3), (5), (11), (16)-(19) it is not hard to get that

$$
\begin{gathered}
\sigma^{2} \geqslant \begin{cases}C_{6}>0, & \tau>5 / 2 ; \\
C_{7}(-\ln (1-\lambda)), & \tau=5 / 2 ; \\
C_{8}(1-\lambda)^{\tau-5 / 2}, & 1<\tau<5 / 2,\end{cases} \\
\sigma^{2} \leqslant \begin{cases}C_{9}>0, & \tau>3 ; \\
C_{10}(1-\lambda)^{\tau-3-\varepsilon}, & 1<\tau \leqslant 3,\end{cases} \\
\left|\varphi^{\prime \prime \prime}(t)\right|= \begin{cases}O(1), & \tau>4 ; \\
O\left((1-\lambda)^{\tau-4-\varepsilon}\right), & 1<\tau \leqslant 4 .\end{cases}
\end{gathered}
$$

The next expression is valid for a sufficiently small $t$ :

$$
\begin{aligned}
\ln \varphi(t)=\left.t(\ln \varphi(t))^{\prime}\right|_{t=o} & +\left.\frac{t^{2}}{2}(\ln \varphi(t))^{\prime \prime}\right|_{t=o}+ \\
& +\frac{t^{3}}{6} Q(t)
\end{aligned}
$$

where $|Q(t)| \leqslant 2 \max _{|u| \leqslant|t|}\left|(\ln \varphi(u))^{\prime \prime \prime}\right|$.

Using (13), (20)-(23) and (A1)-(A5) we get (14). 
According to the inversion formula we represent the probability $\mathbf{P}\left\{\zeta_{N}=k\right\}$ as the integral

$$
\mathbf{P}\left\{\zeta_{N}=k\right\}=\frac{1}{\sigma \sqrt{2 \pi N}} \int_{-\pi \sigma \sqrt{N}}^{\pi \sigma \sqrt{N}} e^{-i z t} \varphi_{N}(t) d t,
$$

where $z=(k-n) /(\sigma \sqrt{N})$. Since

$$
(\sqrt{2 \pi})^{-1} e^{-z^{2} / 2}=(2 \pi)^{-1} \int_{-\infty}^{\infty} e^{-i z t-t^{2} / 2} d t
$$

the difference

$$
R=2 \pi\left[\sigma \sqrt{N} \mathbf{P}\left\{\zeta_{N}=k\right\}-(2 \pi)^{-1 / 2} e^{-z^{2} / 2}\right]
$$

can be rewritten as the sum of four integrals: $R=I_{1}+I_{2}+I_{3}+I_{4}$, where

$$
\begin{gathered}
I_{1}=\int_{-A}^{A} e^{-i z t}\left[\varphi_{N}(t)-e^{-t^{2} / 2}\right] d t, \\
I_{2}=\int_{A<|t|<a \sigma \sqrt{N}} e^{-i z t} \varphi_{N}(t) d t, \\
I_{3}=\int_{a \sigma \sqrt{N} \leqslant|t| \leqslant \pi \sigma \sqrt{N}} e^{-i z t} \varphi_{N}(t) d t, \\
I_{4}=-\int_{A<|t|} e^{-i z t-t^{2} / 2} d t, \\
\text { From }(13) \text { we get that } \\
\begin{array}{c}
\ln \varphi\left(\frac{t}{\sigma \sqrt{N}}\right)=\left\{\begin{array}{l}
(1-\lambda)^{-\tau+} \\
(-\ln (1-\lambda) \\
(1-\lambda)^{3 / 2+}
\end{array}\right. \\
=\frac{t^{3}}{\sigma \sqrt{N}}-\frac{t^{2}}{2 N}+\frac{t}{6 \sigma^{3} N^{3 / 2}} Q \\
\left(\frac{t}{\sigma \sqrt{N}}\right),
\end{array}
\end{gathered}
$$

$$
B(\lambda, \tau)= \begin{cases}(1-\lambda)^{-\tau+4+\varepsilon,} & 5 / 2<\tau \leqslant 4 \\ (-\ln (1-\lambda))(1-\lambda)^{3 / 2+\varepsilon}, & \tau=5 / 2 \\ (1-\lambda)^{3 / 2+\varepsilon}, & 1<\tau<5 / 2\end{cases}
$$

where

$$
|Q(t /(\sigma \sqrt{N}))| \leqslant 2 \max _{|u| \leqslant|t /(\sigma \sqrt{N})|}\left|\ln ^{\prime \prime \prime} \varphi(u)\right| .
$$

In the integration domains of the integral $I_{2}^{\prime} t /(\sigma \sqrt{N}) \rightarrow 0$, then from Lemma 1, (17), (20) and (27) we obtain:

$$
\left|\frac{t}{\left(\sigma^{3} \sqrt{N}\right)} Q\left(\frac{t}{\sigma \sqrt{N}}\right)\right| \leqslant a .
$$

the positive constants $A$ and $a$ will be chosen later. Lemma 2 will be proved if we show that by choosing sufficiently large $n, N$ the difference $R$ can be made arbitrarily small. From (14) we get that $I_{1} \rightarrow 0$. Moreover,

$$
\left|I_{4}\right| \leqslant \int_{A<|t|} e^{-t^{2} / 2} d t
$$

and the integral $I_{4}$ is as small as desired, provided that $A$ is large enough.

Let us estimate the integral $I_{2}$. From (23) and (12) we obtain that for sufficiently small a $\left|\varphi_{N}(t)\right| \leqslant e^{-C_{11} t^{2}}$ as $n / N \rightarrow 1$, therefore the next estimate is true $\left|I_{2}\right| \leqslant \int_{A<|t|} e^{-C_{11} t^{2}} d t$, and the integral $I_{2}$ is small for large enough $A$. From (13), (17) and (20) we obtain the same estimate as $n / N \nearrow \Sigma(1, \tau-1) / \Sigma(1, \tau), \tau>4$.

Let $n / N \nearrow \Sigma(1, \tau-1) / \Sigma(1, \tau), \tau \leqslant 4$. We divide $I_{2}$ into integrals $I_{2}^{\prime}$ and $I_{2}^{\prime \prime}$, where the integration domains are

$$
\{t: A<|t| \leqslant a B(\lambda, \tau) \sigma \sqrt{N}\}
$$

and

$$
\{t: a B(\lambda, \tau) \sigma \sqrt{N}<|t| \leqslant a \sigma \sqrt{N}\},
$$

where
It follows that for small enough $a \quad\left|\varphi_{N}(t)\right| \leqslant$ $\exp \left\{-C_{12} t^{2}\right\}$. Therefore

$$
\left|I_{2}^{\prime}\right| \leqslant 2 \int_{A<|t|} e^{-C_{12} t^{2}} d t
$$

and the integral $I_{2}^{\prime}$ is as small as desired, provided that $A$ is large enough. To estimate the integral $I_{2}^{\prime \prime}$ we expand the function $\Sigma(\lambda z, \tau)$, where $z=$ $e^{i t /(\sigma \sqrt{N})}$ in the Taylor series near the point $z=1$. Then

$$
\begin{gathered}
\varphi(t)=1-(1+o(1)) \Sigma(\lambda, \tau-1) \Sigma^{-1}(\lambda, \tau) \times \\
\times(1-\cos (t /(\sigma \sqrt{N}))-i \sin (t /(\sigma \sqrt{N}))) .
\end{gathered}
$$

Therefore

$$
\left|\varphi_{N}(t)\right| \leqslant\left|\varphi^{N}(t /(\sigma \sqrt{N}))\right| \leqslant
$$




$$
\leqslant \exp \left\{C_{13} N(1-\cos (t /(\sigma \sqrt{N})))\right\} .
$$

Using (27), the conditions (A1) - (A5) and the inequality

$$
\begin{aligned}
1-\cos (t /(\sigma \sqrt{N})) & \geqslant 1-\left(1-C_{14} t^{2} /\left(\sigma^{2} N\right)\right), \\
|t| & <a \sigma \sqrt{N},
\end{aligned}
$$

we can show that

$$
\begin{gathered}
\left|I_{2}^{\prime \prime}\right| \leqslant \int_{a B(\lambda, \tau) \sigma \sqrt{N}}^{\infty} e^{-C_{14} t^{2} / \sigma^{2}} d t \leqslant \\
\leqslant C_{15} \frac{\sigma}{a B(\lambda, \tau) \sqrt{N}} e^{-C_{14} a^{2} B^{2}(\lambda, \tau) N} \rightarrow 0 .
\end{gathered}
$$

Let us consider the integral $I_{3}$. For $\varepsilon \leqslant|t| \leqslant \pi$ the inequality

$$
|\varphi(t)| \leqslant e^{-C_{16}}
$$

is valid. Then under the condition that $n / N \rightarrow 1$ it can be shown that

$$
\varphi(t)=e^{i t}\left(1+\lambda p_{2}\left(e^{i t}-1\right) / p_{1}\right)+o\left(\lambda^{2}\right) .
$$

From this and Lemma 1 we get that for $\varepsilon \leqslant$ $|t| /(\sigma \sqrt{N}) \leqslant \pi$

$$
|\varphi(t /(\sigma \sqrt{N}))| \leqslant e^{-C_{17} \lambda} .
$$

Therefore using relations (12) and (25) it is not hard to see that

$$
\left|I_{3}\right| \leqslant C_{18} \sqrt{n-N} \exp \left\{-C_{19}(n-N)\right\} \rightarrow 0 .
$$

Let $n / N \rightarrow \Sigma(1, \tau-1) / \Sigma(1, \tau)$. From the conditions (A1) - (A5), (21) and (28) we get that

$$
\left|I_{3}\right| \leqslant C_{20} \sigma \sqrt{N} e^{-C_{21} N} \rightarrow 0 .
$$

Thus Lemma 2 is proved.

Let $\varphi_{r}(t)$ be the characteristic function of the random variable $\left(\zeta_{N}^{(r)}-n\right) /(\sigma \sqrt{N})$.

Lemma 3. Let $n, N \rightarrow \infty$. Then uniformly with respect to $t$ in any fixed finite interval the next conclusions are true

1. if $n / N \rightarrow 1,(n-N)^{3} / N^{2} \rightarrow \infty, N P_{r-1} \rightarrow$ $\infty, N P_{r} \rightarrow \gamma$, where $\gamma$ is a nonnegative constant, then for $s=0, \pm 1 \quad \varphi_{r+s}(t) \rightarrow$ $e^{-t^{2} / 2}$

2. if $n / N \rightarrow \Sigma(1, \tau-1) / \Sigma(1, \tau), N P_{r} \rightarrow \gamma$, where $\gamma$ is a positive constant, parameters $\tau, N, n$ are determined by the conditions $(\mathrm{A} 1)-(\mathrm{A} 5)$ then $\varphi_{r}(t) \rightarrow e^{-t^{2} / 2}$.
Proof. From (7) and (10) it is easy to see that

$$
\begin{gathered}
\varphi_{r}(t)= \\
=\exp \left\{-\frac{i t n}{\sigma \sqrt{N}}\right\}\left(1-P_{r}\right)^{-N} \varphi^{N}\left(\frac{t}{\sigma \sqrt{N}}\right) \times \\
\times\left(1-(1+o(1)) \sum_{k=r+1}^{\infty} p_{k}(\lambda) \exp \left\{\frac{i t k}{\sigma \sqrt{N}}\right\}\right)^{N} .
\end{gathered}
$$

It is not hard to get that

$$
\begin{gathered}
\sum_{k=r+1}^{\infty} p_{k}(\lambda) \exp \{t k /(\sigma \sqrt{N})\}= \\
=P_{r}+R(t)
\end{gathered}
$$

where $R(t) \leqslant|t / \sigma \sqrt{N}| \sum_{k=r+1}^{\infty} p_{k}(\lambda) k$.

Let $n / N \rightarrow 1$. It is clear that

$$
N P_{r}=N \sum_{i \geqslant 0} p_{r+i+1}(\lambda)=
$$

$$
=N\left(\sum_{i=0}^{M} p_{r+i+1}(\lambda)+\sum_{i \geqslant M+1} p_{r+i+1}(\lambda)\right),
$$

the positive constant $M$ will be chosen later. For the fixed integer $r$ we get from Lemma 1, (2)-(4) and (15) that

$$
\sum_{i=0}^{M} p_{r+i+1}(\lambda)=\frac{N \lambda^{r+1} h(r+1)}{\Sigma(\lambda, \tau)(r+1)^{\tau}}(1+o(1))
$$

and for large enough $N$

$$
\sum_{i \geqslant M+1} p_{r+i+1}(\lambda)=O\left(\sum_{i \geqslant 0}^{M} p_{r+i+1}(\lambda)\right) .
$$

Therefore

$$
N P_{r}=\frac{N \lambda^{r+1} h(r+1)}{\Sigma(\lambda, \tau)(r+1)^{\tau}}(1+o(1)) .
$$

Using $N P_{r} \rightarrow \gamma$ we obtain that for fixed integer $r$

$$
(\sigma \sqrt{N})^{-1} \sum_{k>r} k p_{k}(\lambda)=o\left(N^{-1}\right) .
$$

As $r \rightarrow \infty$ we can deduce from Lemma 1, (2)-(4) and (15) the relation (33) is valid.

From (2)-(4), (15) and the relation $N P_{r} \rightarrow \gamma$ we can get that as $r \rightarrow \infty$

$(\sigma \sqrt{N})^{-1} \sum_{k>r} k p_{k}(\lambda) \leqslant C_{22} t(r+1) p_{r}(\lambda) /(\sigma \sqrt{N})$. 
Since $N P_{r-1} \rightarrow \infty$ it is not hard to show that $r=o(\sqrt{n-N})$. From this, (31), (35) it follows that

$$
(\sigma \sqrt{N})^{-1} \sum_{k>r} k p_{k}(\lambda)=o\left(N^{-1}\right) .
$$

Therefore, for $n / N \rightarrow 1$ the relation $\varphi_{r}(t) \rightarrow$ $e^{-t^{2} / 2}$ holds.

For $s=1$ we get that $N P_{r+1} \rightarrow 0$. Therefore in this case the assertion of Lemma 3 follows from (30) by substituting $r$ with $r+1$.

Let $s=-1$. By analogy with the estimate (34) we can obtain that as $r \rightarrow \infty$

$$
(\sigma \sqrt{N})^{-1} \sum_{k \geqslant r} p_{k}(\lambda) k \leqslant C_{23} r p_{r}(\lambda) /(\sigma \sqrt{N}) .
$$

Using (15) and the condition $(n-N)^{3} / N^{2} \rightarrow \infty$ the relation (34) follows from this and (2)-(4). By analogy with this estimate for fixed integer $r$ we can get that

$$
\begin{gathered}
\frac{1}{\sigma \sqrt{N}} \sum_{k \geqslant r} p_{k}(\lambda) k= \\
=\frac{\operatorname{trp}_{r}(\lambda)}{\sigma \sqrt{N}} \sum_{k \geqslant r} \lambda^{k-r}\left(\frac{r}{k}\right)^{\tau-1} \frac{h(k)}{h(r)} \leqslant \\
\leqslant C_{24} \frac{\operatorname{trp}_{r}(\lambda)}{\sigma \sqrt{N}}=o\left(\frac{1}{N}\right) .
\end{gathered}
$$

Therefore, as $n / N \rightarrow 1$, the relation $\varphi_{r-1}(t) \rightarrow$ $e^{-t^{2} / 2}$ holds

Let $n / N \nearrow \Sigma(1, \tau-1) / \Sigma(1, \tau)$. Using (2)(4), the properties of the slowly varying function (15), Lemma 1 and (32) we can deduce that

$$
\sum_{i=0}^{M} p_{r+i+1}(\lambda)=p_{r+1}(\lambda) \sum_{i=0}^{M} \lambda^{i}(1+o(1)) .
$$

From (15) it is not hard to get

$$
\sum_{i \geqslant M+1} p_{r+i+1}(\lambda)=o\left(\sum_{i=0}^{M} p_{r+i+1}(\lambda)\right) .
$$

From the condition $N P_{r} \rightarrow \gamma$ it follows that $r \rightarrow \infty$. Then from (32), (36), (37) we get that

$$
N P_{r}=\frac{N \lambda^{r+1} h(r+1)}{\Sigma(\lambda, \tau)(r+1)^{\tau}(1-\lambda)}(1+o(1)) .
$$

Using Lemma 1, (15), (38) and the condition $N P_{r} \rightarrow \gamma$ it is not hard to see that

$$
\frac{t}{\sigma \sqrt{N}} \sum_{k \geqslant r+1} p_{k}(\lambda) k \leqslant C_{25} \frac{t(r+1) p_{r+1}(\lambda)}{\sigma \sqrt{N}(1-\lambda)} \leqslant
$$

$$
\leqslant C_{26} \frac{t(r+1) \gamma}{\sigma N^{3 / 2}} .
$$

From (38) and the condition $N P_{r} \rightarrow \gamma$ it is easy to see that

$$
\frac{N \lambda^{r+1} h(r+1)}{\left(\Sigma(\lambda, \tau)(r+1)^{\tau}(1-\lambda)\right)} \rightarrow \gamma>0 .
$$

Using the conditions (A1)-(A5), (20) and (40) we get that $(r+1) /(\sigma \sqrt{N})=o(1)$. From this and (39) we can obtain that

$$
t(\sigma \sqrt{N})^{-1} \sum_{k \geqslant r+1} p_{k}(\lambda) k=o\left(N^{-1}\right) .
$$

Then the assertion of Lemma 3 follows from (30) and (31).

Lemma 4. Let $n, N \rightarrow \infty$ and one of the following conditions be fulfilled

1. $n / N \rightarrow 1,(n-N)^{3} / N^{2} \rightarrow \infty, N P_{r-1} \rightarrow$ $\infty, N P_{r} \rightarrow \gamma$, where $\gamma$ is a nonnegative constant;

2. $n / N \quad \nearrow \quad \Sigma(1, \tau-1) / \Sigma(1, \tau), N P_{r} \rightarrow$ $\gamma$, where $\gamma$ is a positive constant and parameters $\tau, N, n$ are determined by one of the conditions (A1)-(A5).

Then

$$
\mathbf{P}\left\{\zeta_{N}^{(r)}=k\right\}=\frac{1+o(1)}{\sigma \sqrt{2 \pi N}} \exp \left\{-\frac{(k-n)^{2}}{2 \sigma^{2} N}\right\}
$$

uniformly with respect to integers $k$ such that $(k-n) /(\sigma \sqrt{N})$ lies in any fixed finite interval.

Proof. We follow the scheme of proving Lemma 2. We represent the probability $\mathbf{P}\left\{\zeta_{N}^{(r)}=k\right\}$ as the integral

$$
\mathbf{P}\left\{\zeta_{N}^{(r)}=k\right\}=\frac{1}{2 \pi \sigma \sqrt{N}} \int_{-\pi \sigma \sqrt{N}}^{\pi \sigma \sqrt{N}} e^{-i z t} \varphi_{r}(t) d t,
$$

where $z=(k-n) /(\sigma \sqrt{N})$ and $\varphi_{r}(t)$ is the characteristic function of the random variable $\left(\zeta_{N}^{(r)}-n\right) /(\sigma \sqrt{N})$. Using $(24)$ the difference

$$
R=2 \pi\left[\sigma \sqrt{N} \mathbf{P}\left\{\zeta_{N}^{(r)}=k\right\}-(2 \pi)^{-1 / 2} e^{-t^{2} / 2}\right]
$$

can be rewritten as the sum of four integrals: $R=I_{1}^{(r)}+I_{2}^{(r)}+I_{3}^{(r)}+I_{4}$, where $I_{4}$ is given by (25) and $I_{1}^{(r)}-I_{3}^{(r)}$ are constructed similarly to $I_{1}-I_{3}$ by substituting $\varphi_{r}(t)$ instead of $\varphi_{N}(t)$ in (25). 
From Lemma 3 it follows that $I_{1}^{(r)} \rightarrow 0$. From, (12), (20)-(23) and (30) we get that

$\left|\varphi_{r}(t)\right| \leqslant\left(1-P_{r}\right)^{-N}\left(\exp \left\{C_{27} t^{2} / N\right\}+C_{28} / N\right)^{N}$.

Therefore, $\left|I_{2}^{(r)}\right| \leqslant C_{29} \int_{A}^{\infty} e^{-C_{30} t^{2}} d t$. It is clear that $I_{2}^{(r)}$ can be made arbitrarily small by choosing a sufficiently large $A$.

It is easy to show that we can estimate the integral $I_{3}^{(r)}$ by analogy with $I_{3}$ in Lemma 2. For $I_{4}$ we can use the estimation (26). This completes the proof of Lemma 4.

It is not hard to see that the conclusion of Lemma 4 is true when replacing $\mathrm{r}$ with $r-1$ and $r+1$ as $n / N \rightarrow 1$. In these cases the proofs are similar to the proof of Lemma 4.

From (4) and (7) we have that

$$
\begin{gathered}
m_{r}=\mathbf{E} \tilde{\xi}_{1}^{(r)}=\left(m-r p_{r}(\lambda)\right) /\left(1-p_{r}(\lambda)\right), \\
\sigma_{r}^{2}=\mathbf{D} \tilde{\xi}_{1}^{(r)}=\frac{\sigma^{2}}{\left(1-p_{r}(\lambda)\right)^{2}} \times \\
\times\left(1-p_{r}(\lambda)-\frac{(m-r)^{2}}{\sigma^{2}} p_{r}(\lambda)\right) .
\end{gathered}
$$

Let $\tilde{\varphi}_{r}(t)$ be the characteristic function of the random variable $\left(\tilde{\zeta}_{N}^{(r)}-S m_{r}\right) /\left(\sigma_{r} \sqrt{S}\right)$. By analogy with Lemmas $2-4$ it is not hard to prove the following assertions.

Lemma 5. Let $n, N \rightarrow \infty$ and one of the following conditions be fulfilled

1. $n / N \rightarrow 1, r=1,(n-N)^{2} / N \rightarrow \infty$;

2. $n / N \rightarrow 1, r=2,(n-N)^{2} / N \rightarrow \infty$;

3. $n / N \rightarrow 1, r \geqslant 3, n-N \rightarrow \infty$;

4. $n / N \rightarrow \Sigma(1, \tau-1) / \Sigma(1, \tau)$ and parameters $\tau, N, n$ are determined by one of the conditions (A1)-(A5).

Then for $S=N\left(1-p_{r}(\lambda)\right)(1+o(1))$

$$
\tilde{\varphi}_{r}(t) \rightarrow e^{-t^{2} / 2}
$$

uniformly with respect to $t$ in any fixed finite interval.

Lemma 6. Under the conditions of Lemma 5 for $S=N\left(1-p_{r}(\lambda)\right)(1+o(1))$

$$
\mathbf{P}\left\{\tilde{\zeta}_{S}^{(r)}=k\right\}=\frac{1}{\sigma_{r} \sqrt{2 \pi S}} e^{-z^{2} / 2}(1+o(1))
$$

uniformly with respect to integers $k$ such that $z=\left(k-S m_{r}\right) /\left(\sigma_{r} \sqrt{S}\right)$ lies in any fixed finite interval.

\section{Proofs of THEOREMS}

We are now ready to prove Theorems $1-4$. Using Lemmas 2 and 4 we obtain

$$
\mathbf{P}\left\{\zeta_{N}^{(r)}=n\right\} / \mathbf{P}\left\{\zeta_{N}=n\right\} \rightarrow 1 .
$$

The assertion of Theorem 1 follows from $N P_{r} \rightarrow \gamma, N P_{r-1} \rightarrow \infty,(8),(33)$ and (42). The assertion of Theorem 2 follows from (8), (38) and (42).

According to the normal approximation of the binomial distribution under the condition $N p_{r}(\lambda)\left(1-p_{r}(\lambda)\right) \rightarrow \infty$ Theorem 3 follows from (9), (41) and Lemmas 2, 6.

Using Poisson approximation of the binomial distribution as $p_{r}(\lambda) \rightarrow 0$, Lemmas 2, 6 and relations (9), (41) we can obtain the assertion of Theorem 4 .

The study was carried out under state order to the Karelian Research Centre of the Russian Academy of Sciences (Institute of Applied Mathematical Research KarRC RAS) and supported by the Russian Foundation for Basic Research, grant 16-01-00005a.

\section{REFERENCES}

1. Bateman H., Erdelyi A. Higher trancedendental functions. Vol. 1. New York; Toronto; London: Megraw-hill book company, inc, 1953. 317 p.

2. Cheplyukova I. A. On limit distributions of vertex degrees in a configuration graph. Trans. KarRC RAS. 2015. No. 10. P. 123-130. doi: 10.17076/mat138 (in Russian)

3. Faloutsos M., Faloutsos P., Faloutsos Ch. On power-law relationships of the internet topology. Computer Communications Rev. 1999. Vol. 29. P. 251-262. doi: 10.1145/316194.316229

4. Ibragimov I. A., Linnik $Y u$. V. Independent and stationary sequences of random variables. Groningen: Wolters-Noordhoff, 1971. 443 p.

5. Kolchin V. F. Random Mapping. New York: Springer, 1986. 207 p.

6. Newman M. E. Y., Strogatz S. H., Watts D. Y. Random graphs with arbitrary degree distribution and their applications.Physical Review E. 2001. E 64, 026118. doi: 10.1103/PhysRevE.64.026118

7. Pavlov $Y u$. On the limit distributions of the vertex degrees of conditional Internet graphs. Discrete Mathematics and Applications. 2009. Vol. 19, iss. 4. P. 349-359. doi: 10.1515/dma.2009.023

8. Pavlov $Y u$. On conditional Internet graphs whose vertex degrees have no mathematical expectation. Discrete Mathematics and Applications. 2010. Vol. 20, iss. 5-6. P. 509-524. doi: 10.1515/dma.2010.031 
9. Pavlov Yu., Cheplyukova I. Random graphs of Internet type and the generalized allocation scheme. Discrete Mathematics and Applications. 2008. Vol. 18, iss. 5. P. 447-464. doi: 10.1515/dma.2008.033

10. Pavlov Yu., Dertishnikova E. On the limit distribution of the maximum vertex degree of random graph of the Internet type. Trans. KarRC RAS. 2010. No. 3. P. 59-65 (in Russian).

11. Reittu H., Norros I. On the power-law random graph model of massive data networks. Performance Evaluation. 2004. Vol. 55. P. 3-23. doi: 10.1016/S0166-53/6(3)00097-x

Received January 31, 2018

\section{CONTRIBUTORS:}

Pavlov, Yury

Institute of Applied Mathematical Research, Karelian Research Centre,

Russian Academy of Sciences

11 Pushkinskaya St., 185910 Petrozavodsk,

Karelia, Russia

e-mail: pavlov@krc.karelia.ru

tel.: (8142) 781218

эл. почта: pavlov@krc.karelia.ru

тел.: (8142) 781218

\section{Чеплюкова Ирина Александровна}

старший научный сотрудник, к. ф.-м. н., доцент

Институт прикладных математических исследований

КарНЦ РАН, Федеральный исследовательский центр

«Карельский научный центр РАН»

ул. Пушкинская, 11, Петрозаводск,

Республика Карелия, Россия, 185910

эл. почта: chia@krc.karelia.ru

тел.: (8142) 781218

\section{Cheplyukova, Irina}

Institute of Applied Mathematical Research, Karelian Research Centre,

Russian Academy of Sciences

11 Pushkinskaya St., 185910 Petrozavodsk,

Karelia, Russia

e-mail: chia@krc.karelia.ru

tel.: (8142) 781218 